\title{
Optimal Timing and Antibiotic Prophylaxis in Periprosthetic Joint Infection (PJI): Literature Review and World Consensus (Part Three)
}

\author{
Javad Parvizi ${ }^{1, *}$; Mohammad Ghazavi ${ }^{2,3}$; Members of Pre-op Antibiotics Committee of the \\ Consensus Meeting ${ }^{4}$ \\ ${ }_{1}^{1}$ Rothman Institute, Thomas Jefferson University, Philadelphia, PA, USA \\ ${ }^{2}$ Scarborough Hospital, Toronto, Ontario, Canada \\ ${ }^{3}$ Shafa Orthopedic Hospital, Iran University of Medical Sciences, Tehran, IR Iran \\ 4 William Arnold MD PhD; Katherine Belden MD; Goran Bicanic; Stefano Bini MD; Fabio Catani MD; Jiying Chen MD PhD; Mohammad Ghazavi MD FRCSC; Karine M. Godefroy MD; Erik \\ Hansen MD; Paul Holham MD; Hamid Hosseinzadeh MD; Kang II Kim MD; Klaus/Kirketerp-Moller MD; Lars Lidgren MD PhD; Jian Hao|Lin MD; Jess H Lonner MD; Christopher Moore \\ MD; Panayiotis Papagelopoulos MD; Lazaros Poultsides MD MSc PhD; Khalid Saleh MD MSC FRCSC MHCM; Julia V Salmon MD; Edward Schwarz PhD; Randi Silibovsky MD (US); Jose \\ Stuyck MD; Markus Vogt MD (International); Annette W Dahl MD; Koji Yamada MD \\ *Corresponding author: Javad Parvizi, Rothman Institute, Thomas Jefferson University, Philadelphia, PA, USA. Tel: +1-2673397813, Fax: +1-2155030580, E-mail: parvizi@aol.com
}

Received: December 3, 2014; Revised: January 2, 2015; Accepted: February 1, 2015

Context: There is a need to find the situations that Vancomycin should be used as antibiotic prophylaxis in hip and knee arthroplasty. There are also situations that might need different antibiotic prophylaxis protocol; such as abnormal urinary screening test or indwelling urinary catheter and previous joint infection.

Evidence Acquisition: Delegates in workgroup 3 of the consensus meeting on periprosthetic joint infection (PJI) reviewed English literature for relevant articles. 62 of 221 articles were relevant to the six following questions regarding perioperative antibiotic prophylaxis to prevent PJI.

Results:Vancomycin should be considered for patients who are current Methicillin resistant Staphylococcus aureus (MRSA) carriers or have anaphylactic allergy to penicillins. Routine use of vancomycin for preoperative prophylaxis is not recommended. Routine prophylactic use of dual antibiotics is not recommended. The presence of urinary tract symptoms should trigger urinary screening prior to total joint arthroplasty (TJA). Asymptomatic patients with bacteriuria may safely undergo TJA provided that routine prophylactic antibiotics are administered. Patients with acute urinary tract infections (UTI) need to be treated prior to elective arthroplasty. The type of preoperative antibiotic administered to a patient with prior septic arthritis or PJI should cover the previous infecting organism of the same joint. In these patients, we recommend the use of antibiotic-impregnated cement, if a cemented component is utilized. There is no evidence to support the continued use of postoperative antibiotics when urinary catheter or surgical drains are in place. Urinary catheters and surgical drains should be removed as soon as safely possible.

Conclusions: Recommendations for choice of perioperative antibiotic prophylaxis in hip and knee arthroplasty were provided based on evidences in the literature and consensus of expert delegates from consensus meeting.

Keywords: Infection; Periprosthetic; Joints; Arthroplasty

\section{Context}

Decision making in selecting the best choice of antibiotic prophylaxis for periprosthetic joint infection (PJI) is a challenge for all arthroplasty surgeons. There are special situations where routine use of first-generation cephalosporins is not sufficient, appropriate, or even is contraindicated. In these situations Vancomycin could be used as an alternative. There might be a need for different antibiotic prophylaxis protocols such as abnormal urinary screening test, indwelling urinary catheter and previous joint infection.

\section{Evidence Acquisition}

From November 2012 till August 2013, 400 delegates from all over the world formed 15 workgroups to review the current literature and find high level evidence for all issues related to PJI. Workgroup No.3 (authors) was assigned to review current literature on perioperative antibiotics. The goal was to find answers and recommendations for more than 264 questions based on the high level evidence if present or reach to a consensus when there is a lack of high level evidence.

Copyright ( 2015 , Iran University of Medical Sciences. This is an open-access article distributed under the terms of the Creative Commons Attribution-NonCommercial 4.0 International License (http://creativecommons.org/licenses/by-nc/4.0/) which permits copy and redistribute the material just in noncommercial usages, provided the original work is properly cited. 
After 10 months of hard work by delegates from 58 countries and 100 societies, relevant publications reviewed, communications exchanged and finally a draft was prepared to be presented for vote at the final meeting on 1st of August 2013. The draft included recommendations for management on the basis of high level of evidence if present. Otherwise the cumulative wisdom of 400 delegates from 58 countries and over 100 societies used to reach consensus about practices lacking higher level of evidence.

\section{Results}

Question 6: What are the indications for administration of vancomycin?

Consensus: Vancomycin should be considered for patients who are current MRSA carriers or have anaphylactic allergy to penicillins.

Consideration should be given to screening high risk patients such as:

- Patients in regions with a high prevalence of MRSA.

- Institutionalized patients (nursing home residents, dialysis-dependent patients, and those who have been in the intensive care unit).

- Healthcare workers.

Delegate Vote: agree: 93\%, disagree: 7\%, abstain: 0\% (strong consensus)

Justification: The AAOS recommendation for the use of IV antibiotic prophylaxis in primary

TJA, recommendation 2, states that vancomycin may be used in patients with known colonization with MRSA or in facilities with recent MRSA outbreaks (1). Similarly, the consensus 65 position of the medicare national surgical infection prevention project hosted the surgical infection prevention guideline writers workgroup (SIPGWW) meeting was that for patients with known MRSA colonization, vancomycin should be considered the appropriate antimicrobial agent for prophylaxis. Additionally, the society for healthcare epidemiology of America recently recommended routine surveillance cultures at the time of hospital admission for patients at high risk for carriage of MRSA (2).

Question 7: Is there evidence to support the routine use of vancomycin for preoperative prophylaxis?

Consensus: No. Routine use of vancomycin for preoperative prophylaxis is not recommended.

Delegate Vote: agree: 93\%, disagree: 6\%, abstain: $1 \%$ (strong consensus)

Justification: Current data suggest that the role of vancomycin in orthopedic surgery prophylaxis should be limited. There is ample evidence that vancomycin is inferior against methicillin-sensitive strains of staphylococcal species when compared to cephalosporin and penicillinase-resistant penicillin $(3,4)$.

Several systematic analyses concluded that no clear benefit in clinical or cost effectiveness has been demonstrated for the routine use of vancomycin compared with cephalosporin for prophylaxis. However, most of these studies were conducted before the increasing prevalence of MRSA and may not accurately reflect the current environment. In some hospitals, community associated MRSA (CA-MRSA) strains are now responsible for a significant portion of surgical site infections (SSIs) $(5,6)$.

However, there is no consensus about what constitutes a high prevalence of methicillin resistance and no evidence that routine use of vancomycin for prophylaxis in institutions with perceived high risk of MRSA infection results in fewer SSIs than the use of a cephalosporin.

Although two randomized controlled trials (RCTs) have been conducted in institutions with a high MRSA prevalence, the differences in SSI rates and outcomes were conflicting. Similarly, several studies have utilized decision analysis models to calculate MRSA prevalence thresholds for which vancomycin would have clinical benefit and be more cost-effective than cephalosporin for surgical prophylaxis (7).

However, these studies all suffer from the lack of randomization to provide baseline probabilities for the clinical effectiveness of each treatment at different rates of MRSA prevalence.

While there is a growing body of evidence to support the routine use of vancomycin for preoperative prophylaxis, this should be tempered by the fact that there is an increasing threat of colonization and infection with vancomycin-resistant enterococci (VRE) 56 and an increased prevalence of MRSA strains with reduced susceptibility to vancomycin $(8,9)$.

The choice of drug prophylaxis should take into account the antibiotic resistance patterns in hospital systems. In a recent study by Fulkerson et al. (10), the susceptibilities of S. epidermidis and S. aureus to cefazolin at two high-volume academic centers in New York and Chicago were only $44 \%$ and $74 \%$, respectively. Of the most common organisms infecting patients undergoing TJA at these hospitals, $26 \%$ to $56 \%$ were resistant to the standard recommended prophylactic agent. Thirty-three of the 194 infections were diagnosed within a month after the surgery. Of these, 8 were due to $S$. epidermidis and 16 were due to S. aureus. Of these, only 2 of the 8 (25\%) of the $S$. epidermidis infections and 11 of the 16 (69\%) of the S. aureus infections were sensitive to cefazolin. However, these infections were $100 \%$ susceptible to vancomycin.

In a study of deep infections following hip and knee arthroplasty over a 15-year period at the

Royal orthopedic hospital and Queen Elizabeth hospital in England, 22 of 75 hip and knee infections (29\%) were caused by microorganisms that were resistant to the antibiotic used for prophylaxis (cefuroxime). These included all 3 MRSA infections, all 3 Pseudomonas aeruginosa infections, and 11 coagulase-negative Staphylococcus infections (11,12). Wiesel and Esterhai (13) recommend administration of vancomycin in institutions where the prevalence of MRSA is greater than $10 \%$ to $20 \%$. In a hospital with a high prevalence of MRSA, Merrer et al. (14) conducted a prospective, observational study comparing 
Parvizi J et al.

the incidence of SSI after vancomycin or cefazolin prophylaxis before femoral neck fracture surgery, as well as the impact of antibiotic prophylaxis on the emergence of VRE and Staphylococcus aureus. The authors found no significant difference in the rate of SSI, as a total of $8(3 \%)$ occurred, $4 \%$ in the cefazolin group and $2 \%$ in the vancomycin group $(P=0.47)$. At one week after surgery, there were a total of 6 patients (2\%) who had hospital-acquired MRSA, corresponding to $0.7 \%$ in the cefazolin group and $5 \%$ in the vancomycin group $(\mathrm{P}=0.04)$, none of which were resistant to glycopeptides. Additionally, 3 patients (1\%) acquired VRE, all of which were in the cefazolin group $(\mathrm{P}=0.27)(14,15)$.

Cranny et al. (15) used a combination of systematic reviews and economic modeling in order to answer questions about whether there is a level of MRSA prevalence at which a switch from non-glycopeptide to glycopeptide antibiotics for routine prophylaxis is indicated in surgical environments with a high risk of MRSA infection. The effectiveness reviews identified 16 RCTs with a further 3 studies included for adverse events only. They found no evidence to support that glycopeptides are more effective than non glycopeptides in preventing SSI. Most of the trials did not report either the baseline prevalence of MRSA at the participating surgical units or MRSA infections as an outcome. The cost-effectiveness review included 5 economic evaluations of glycopeptide prophylaxis. Only one study incorporated health-related quality of life and undertook a cost-utility analysis. In conclusion, the authors indicate that there is currently insufficient evidence to determine whether there is a threshold prevalence of MRSA at which switching from non-glycopeptide to glycopeptide antibiotic prophylaxis might be cost effective (15).

Bolon et al. (16) performed a meta-analysis of 7 RCTs published in the cardiothoracic surgery literature that compared SSIs in subjects receiving glycopeptide prophylaxis with those who received $\beta$-lactam prophylaxis. While neither agent proved to be superior for prevention of the primary outcome, occurrence of SSI at 30 days (RR $1.14,95 \%$ CI 0.91 - 1.42), vancomycin prophylaxis was superior for the prevention of SSI caused by methicillin-resistant gram-positive bacteria (RR, $0.54 ; 95 \% \mathrm{CI} 0.33$ - 0.90) at 30 days after surgery.

The AAOS recommendations for the use of IV antibiotic prophylaxis in primary TJA, recommendation 2, states that vancomycin may be used in patients with known colonization with MRSA or in facilities with recent MRSA outbreaks. The hospital infection control practices advisory committee guideline also suggests that a high frequency of MRSA infection at an institution should influence the use of vancomycin for prophylaxis but acknowledges that there is no consensus about what constitutes a high prevalence of methicillin resistance (17).

Two prospective RCTs have evaluated antibiotic prophylaxis in hospitals with a high prevalence of MRSA. Tacconelli et al. (18) randomized patients undergoing surgery for cerebrospinal shunt placement to receive either vancomycin or cefazolin. The prevalence of MRSA in 2001 for a 1700-bed university hospital was reported as one new case of MRSA infection per 100 hospital admissions. Shunt infections developed in $4 \%$ of patients receiving vancomycin (4/88) and $14 \%$ receiving cefazolin (12/88, RR, 0.22; 95\% CI 0.11-0.99, $\mathrm{P}=0.03)$. The infecting pathogen was MRSA in 2 of 4 patients (50\%) receiving vancomycin and 9 of $12(75 \%)$ patients receiving cefazolin (18). Finkelstein et al. (19) randomized 855 patients undergoing cardiothoracic surgery to either a vancomycin or cefazolin group. The prevalence of new cases of MRSA infection in the cardiac surgery ward was reported to be 3.0 and 2.6 per 100 admissions in 1995 and 1996 respectively. The overall rates of SSI were similar in both groups (9.5\% for vancomycin and $9.0 \%$ for cefazolin). A trend toward more methicillin-resistant gram-positive infections was observed in the cefazolin group ( $4.2 \%$ vs. $2.0 \% ; \mathrm{P}=0.09$ ), while more methicillin-sensitive Staphylococcus infections were seen in patients receiving vancomycin (3.7\% vs. $1.3 \% ; \mathrm{P}=0.04)(19)$.

Three other clinical studies have used pre- and postintervention periods to assess the effect of switching to vancomycin for surgical prophylaxis in patients undergoing cardiothoracic or orthopedic surgery. Garey et al. (20) demonstrated that a change from cefuroxime to vancomycin prophylaxis decreased the average monthly SSI rate by 2.1 cases/100 coronary artery bypass graft (CABG) procedures when compared with patients undergoing cardiac valve replacement surgery. This was attributed to a lower rate of infections caused by MRSA and CNS during this 4-year study of nearly 6,500 patients. Similarly, Spelman et al. (21) reported a decrease in SSI rates from $10.5 \%$ to $4.9 \%(\mathrm{P}<0.001)$ after switching the antibiotic prophylaxis regimen from cefazolin to vancomycin plus rifampin in 1,114 CABG procedures. This was attributed to a decrease in the incidence of MRSA infections from $67 \%$ during the one year pre-intervention period to $0 \%$ in the one year post-intervention period. Smith et al. (22) retrospectively reviewed total and MRSA PJI in 5,036 primary TJAs as well as the cure rate of PJI in a 2 year preintervention period when cefazolin was the antibiotic prophylaxis of choice to the 2 year post intervention period when vancomycin was the antibiotic prophylaxis of choice. They found that with the use of vancomycin the total rate of PJI was significantly reduced $(1.0 \%$ vs. $0.5 \%, P=0.03)$ and the rate of MRSA PJI was also reduced ( $0.23 \%$ vs. $0.07 \%, \mathrm{P}=0.14$ ). Furthermore, PJIs were more successfully treated with irrigation and debridement only, not requiring antibiotic spacers (76.9\% vs. $22.2 \%, \mathrm{P}=0.002)$.

A study published on Australian Surveillance Data (Victorian Healthcare Associated Surveillance System) of over 20,000 cardiac and arthroplasty procedures identified 1,610 case in which vancomycin was administered as compared to 20,939 cases in which a $\beta$-lactam was used. The adjusted OR for an SSI with methicillin-sensitive Staphylococcus aureus (MSSA) was 2.79 (95\% CI 1.6 - 4.9) when vancomycin prophylaxis was administered $(\mathrm{P}<0.001)$, whereas 
the unadjusted OR for an SSI with MRSA was 0.44 (OR 0.19 - 1.004; $\mathrm{P}=0.05)(23)$.

Several recent studies have developed decision analysis models to determine the threshold of

MRSA prevalence at which vancomycin would minimize the incidence and cost of SSI. For CABG surgery, the authors of two studies have recommended a MRSA prevalence threshold of $3 \%$ among infections caused by $S$. aureus (24-26). Miller et al. (27) suggested that lower rates of MRSA prevalence (e.g. $3 \%$-10\%) were within the error of their model and that surgical prophylaxis with vancomycin would have a modest effect in reducing the incidence of SSI. For vascular surgery, a MRSA prevalence of $50 \%$ was suggested before a $\beta$-lactam agent is replaced with vancomycin for surgical prophylaxis. The authors also suggested that an aminoglycoside should be added to the prophylactic regimen once the prevalence of MRSA reaches $10 \%$, which is in agreement with the recent guidelines from the British society of antimicrobial chemotherapy (28). Elliot et al. (29) developed an economic model to explore the cost-effectiveness of vancomycin and/or cephalosporin for surgical prophylaxis in patients undergoing THA. Vancomycin was recommended when the rate of MRSA SSIs is $0.15 \%$ and the rate of non- MRSA SSIs is $0.1 \%$, or when the rate of MRSA infections is $0.2 \%$ and the rate of other infections is $>0.2 \%$. Each of these decision analysis studies noted that their biggest limitation was the lack of available evidence from RCTs, with a high prevalence of MRSA infections as one of the most important factors that influenced modeling assumptions.

Question 8: Is there a role for routine prophylactic use of dual antibiotics (Cephalosporins and aminoglycosides or cephalosporins and vancomycin)?

Consensus: Routine prophylactic use of dual antibiotics is not recommended.

Delegate Vote: agree: $85 \%$, disagree: 14\%, abstain: $1 \%$ (strong consensus)

Justification: Clinical studies have used pre- and postintervention periods to assess the effect of switching to vancomycin for surgical prophylaxis in patients undergoing cardiothoracic surgery. Walsh et al. (30) implemented a comprehensive MRSA bundle program in which vancomycin was added to the routine cefazolin prophylaxis regimen for patients who tested positive for nasal MRSA carriage. Other components of the program included decolonization of all cardiothoracic staff who screened positive for nasal MRSA, application of nasal mupirocin ointment for 5 days in all patients starting one day before surgery, application of topical mupirocin to exit sites after removal of chest and mediastinal tubes, and rescreening of patients for MRSA colonization at the time of hospital discharge. This program resulted in a significant reduction in the SSI rate $(2.1 \%$ to $0.8 \%, \mathrm{P}<0.001)$ as well as a $93 \%$ reduction in postoperative MRSA wound infections (from 32 infections/2,767 procedures during the 3 -year pre-intervention period to 2 infections/2,496 procedures during the 3-year post-intervention period) (30).
Dhadwal et al. (31) conducted a double-blind RCT to compare the efficacy of a 48 hour, weight based dosing of vancomycin plus gentamicin and rifampin versus a 24 hour cefuroxime regimen for antibiotic prophylaxis of sternal wound infections in a high-risk group of patients undergoing CABG surgery. The infection rates significantly decreased from $23.6 \%$ (25/106) in the cefuroxime group to $8.4 \%(8 / 95)$ in the combination vancomycin group $(\mathrm{P}=$ 0.004). Patrick et al. (32) conducted an RCT to compare cefazolin and combinations of cefazolin and either vancomycin or daptomycin in 181 low-risk patients undergoing vascular surgery. Only 6 postoperative MRSA infections were reported ( 2 in the cefazolin group, 4 in the vancomycin plus cefazolin group, and 0 in the daptomycin plus cefazolin group), making the interpretation of the differences between antibiotic regimens difficult.

Sewick et al. (33) retrospectively reviewed 1,828 primary TJAs that received either a dual antibiotic regimen of cefazolin and vancomycin or received cefazolin alone in order to determine the rate of SSI as well as the microbiology of subsequent SSI. There was a total of 22 SSIs (1.2\%) with no significant difference in the infection rate between the dual antibiotic prophylaxis group compared to the single antibiotic regimen ( $1.1 \%$ and $1.4 \%$ respectively, $\mathrm{P}$ $=0.636$ ), while the prevalence of subsequent MRSA infection was significantly lower $(0.002 \%$ vs. $0.08 \%, P=0.02)$.

Ritter et al.(34) administered a single prophylactic dose of vancomycin and gentamicin in a cohort of 201 consecutive TJA patients and documented bactericidal blood concentrations during and for 24 hours after surgery with no postoperative infections.

Elliot et al.(29) developed an economic model to explore the cost effectiveness of vancomycin and/or cephalosporin for surgical prophylaxis in patients undergoing THA. Combination therapy (such as vancomycin plus a cephalosporin) was recommended when the rate of MRSA SSIs is $0.25 \%$ and the rate of non-MRSA SSIs is $0.2 \%$ ).

Thus, based on the available literature, this workgroup feels that dual antibiotics may be utilized to allow broad coverage in institutions or regions where there is a high rate of MRSA infection for which prophylactic vancomycin use is deemed appropriate under question 6 above (22).

Question 9: What should be the antibiotic of choice for patients with abnormal urinary screening and/or an indwelling urinary catheter?

Consensus: The presence of urinary tract symptoms should trigger urinary screening prior to TJA. Asymptomatic patients with bacteriuria may safely undergo TJA provided that routine prophylactic antibiotics are administered. Patients with acute urinary tract infections (UTI) need to be treated prior to elective arthroplasty.

Delegate Vote: agree: $82 \%$, disagree: $12 \%$, abstain: $6 \%$ (strong consensus)

Justification: There is sparse literature on the risk of deep joint infection in patients with abnormal perioperative urinalysis. While several case reports in the $1970 \mathrm{~s}$ linked postoperative UTIs to PJI, $(35,36)$ the literature 
Parvizi J et al.

supporting the correlation between preoperative UTIs and PJI following TJA is inadequate (37). Only 3 studies have directly addressed the relationship between preoperative bacteriuria and PJI following TJA, none of which observed a positive correlation $(38,39)$. To our knowledge there are no studies of patients with symptomatic UTI undergoing TJA with routine perioperative prophylactic antibiotics. There is no evidence either in support of or against proceeding with surgery in this cohort of patients. The presence of UTI symptoms should serve as a preliminary screening tool for surgical clearance of the TJA candidate. Symptoms can then be classified as either irritative or obstructive. Irritative symptoms (such as dysuria, urgency, or frequency) may or may not be related to bacteriuria and a noncentrifuged clean catch midstream urine sample should be evaluated for white blood cells (WBCs) in these patients. In patients with $>104 \mathrm{WBC} / \mathrm{mL}$, a bacterial count and culture should be obtained and in patients with $>4 \mathrm{WBC} /$ high power field and bacterial count $>103 / \mathrm{mL}$, surgery should be postponed until an appropriate course of microbe-specific antibiotics is administered and repeat urinalysis is obtained. On the other hand, asymptomatic patients with bacteriuria may safely undergo TJA provided routine prophylactic antibiotics are administered. Patients with obstructive symptoms should undergo urologic evaluation before arthroplasty, as postoperative urinary retention has been shown to be a risk factor for PJI (40-42).

In a prospective, multicenter study of 362 knee and 2,651 hip arthroplasty cases, the authors reported a deep joint infection rate of $2.5 \%$ for knee and $0.64 \%$ for hip cases at one year follow up.

While univariate analysis showed no association between deep joint infection and preoperative UTI $(>105$ $\mathrm{CFU} / \mathrm{mL}$ ), multivariate regression analysis indicated that postoperative UTI increased the risk of hip PJI (39).

of 1,934 surgical cases (1,291 orthopedic surgeries) performed at a Veterans administration hospital, a preoperative urine culture was obtained in $25 \%$ (489) of cases. Of these, bacteriuria was detected in 54 (11\%) patients, of which only 16 received antimicrobial drugs. The incidence of SSI was similar between those with bacteriuria and those without ( $20 \%$ vs. $16 \%, \mathrm{P}=0.56$ ), while the rate of postoperative UTI was more frequent among patients with bacteriuria than those without ( $9 \%$ vs. $2 \%, \mathrm{P}=0.01$ ). Among the 54 patients with a positive urinary culture, treated and untreated patients were compared. Unexpectedly, a greater proportion of treated patients developed an SSI ( $45 \%$ vs. $14 \%, \mathrm{P}=0.03)$. This effect was greatest among patients with high count bacteriuria (>105 CFU/mL), with SSI occurring in 4 of $8(50 \%)$ of treated vs. 10 $15(7 \%)$ of untreated (P = 0.03 ). These results led the authors to conclude that in this system preoperative urinary cultures were inconsistently ordered and that when they were, they were rarely positive for bacteriuria. Even when bacteriuria was detected, it was usually not treated. The authors noted that treating bacteriuria associated with SSI is likely confounded by fac- tors that contributed to the initial decision to administer antimicrobials in the first place (43).

A retrospective study of 274 THAs found that 5 patients with PJI had perioperative UTIs. However, the same organism was isolated from the urinary tract and hip in only 3 patients. Of these, only one had a documented preoperative urinalysis (44). A retrospective analysis of 277 patients (364 TJAs) showed that 35 patients had evidence of preoperative or perioperative UTI with colony counts greater than $105 \mathrm{CFU} / \mathrm{mL}$ on preoperative clean-catch urine specimens. Only 3 patients (1.1\%) developed joint infections at 9,19 , and 45 months respectively, and none was thought to be due to perioperative UTI.87 Another retrospective analysis found 57 (55 asymptomatic, 2 symptomatic) of 299 arthroplasty patients had bacteriuria on admission. Twenty of the 57 patients went to surgery before the routine culture results were available, but postoperatively received appropriate antibiotics for treatment of the UTI. Another 18 patients underwent surgery during their treatment course for preoperatively-diagnosed UTI, while the other 19 patients completed an appropriate antibiotic course prior to surgery. None of the patients developed a PJI, which led the authors to conclude that a treatment course of antibiotics can be implemented at any time perioperatively once culture data are obtained (40).

The incidence of bacteriuria rises from $0.5 \%$ to $1 \%$ for a single in-and-out catheterization, $10 \%$ to $30 \%$ for catheters in place for up to 4 days, and up to $95 \%$ for catheters in place for 30 days or more $(45,46)$.

Question 10: Should the preoperative antibiotic choice be different in patients who have previously been treated for another joint infection?

Consensus: The type of preoperative antibiotic administered to a patient with prior septic arthritis or PJI should cover the previous infecting organism of the same joint. In these patients, we recommend the use of antibiotic-impregnated cement, if a cemented component is utilized.

Delegate Vote: agree: $84 \%$, disagree: 10\%, abstain: 6\% (strong consensus)

Justification: There is no evidence that septic arthritis or a PJI can be completely cured. Jerry et al. (47) conducted a study of 65 patients who underwent TKA and had a history of prior sepsis or osteomyelitis around the knee. They reported rates of deep PJI of $4 \%$ and $15 \%$ respectively. Lee et al. (48) studied a consecutive series of 20 primary TKAs in 19 patients with a history of prior septic arthritis or osteomyelitis around the knee. They performed a preoperative workup to evaluate for infection that included serologies and plain radiographs in all patients, while 8 patients additionally had tagged WBC scans and 7 patients had a knee aspiration. Intraoperatively, frozen section for evidence of acute inflammation was used to guide decisions on whether the procedure was done as a single or staged procedure. All TKA components were implanted with antibiotic cement containing $1 \mathrm{~g}$ of vancomycin and $1.2 \mathrm{~g}$ of tobramycin/batch of Simplex bone cement. Of the 
17 patients with a minimum of 2 years follow-up, only one developed a PJI approximately 3.5 years from the index arthroplasty. Of note, this was one of the two patients that had been treated in a staged manner and additionally had immunosuppressive comorbidities, including rheumatoid arthritis, insulin-dependent diabetes mellitus, and was taking daily doses of prednisone (48).

Larson et al.(49) performed a retrospective matched case control study to review the clinical results of 19 patients who underwent TKA after infected tibial plateau fractures, comparing them to 19 control subjects matched for age, gender, and arthroplasty year, who underwent TKAs for tibial 74 plateau fractures without a history of infection. Of the 19 case patients, 13 underwent one-stage TKA, while the remainder underwent a staged TKA with either an antibiotic spacer or debridement and intravenous antibiotic therapy. Antibiotic cement was used in the majority of patients. Previously infected knees were 4.1 times more likely to require additional procedures for complications compared with knees with no previous infection (95\% CI $1.2-18.3, \mathrm{P}=0.02$ ). The 5 year infection-free survival was $73 \%$, $10 \%$ in the case group compared with $100 \%$ in the control group $(\mathrm{P}=0.023)$. The authors recommended that in patients at high risk less than one year since active evidence of infection, a two-stage TKA be performed, with antibiotic therapy and a 4 to 6 week delay between procedures (49).

Question 11: Should postoperative antibiotics be continued while a urinary catheter or surgical drain remains in place?

Consensus: No. There is no evidence to support the support the continued use of postoperative antibiotics when urinary catheter or surgical drains are in place. Urinary catheters and surgical drains should be removed as soon as safely possible.

Delegate Vote: agree: 90\%, disagree: 7\%, abstain: 3\% (strong consensus)

Justification: Short-term use of an indwelling catheter after surgery reduces the incidence of urinary retention and bladder over-distension without increasing the rate of UTI and is therefore common practice in many hospitals (50). However, it has been shown that there is an increased risk of UTIs when a catheter is employed for more than 48 hours $(51,52)$. Urinary retention as well as catheterization can both lead to bacteriuria, (52-54) which increases the risk of deep PJI from 3 to 6 times $(38,39,55,56)$.

Literature in the field of surgical oncology demonstrates that bacterial colonization of surgical drains used in breast and axillary procedures is a significant risk factor for the development of SSI and the microorganisms that caused SSIs were the same as those that colonized the drainage tube in $83 \%$ of cases (57). Other studies have demonstrated that there is an association between longer duration of drain use and increased incidence of SSI (58).

The AAOS recommendations for the use of IV antibiotic prophylaxis in primary TJA, recommendation 3, states that the duration of prophylactic antibiotic administration should not exceed the 24 hour postoperative period.
Prophylactic antibiotics should be discontinued within 24 hours of the end of surgery. The medical literature does not support the continuation of antibiotics until all drains or catheters are removed and provide no evidence of benefit when they are continued past 24 hours.

Colonization of drains by skin organisms can certainly occur, but in only $10 \%$ of cases with positive drain tip culture does overt infection develop (59). Michelson et al. (50) conducted RCT of 100 TJA patients using two methods of bladder management: short term ( $<24$ hour) indwelling catheters and intermittent catheterization. All patients received the same perioperative cefazolin prophylaxis. The authors reported a lower incidence of urinary retention in the indwelling catheter group (27\% vs. $52 \%, \mathrm{P}<0.01)$ and a lower rate of bladder distension $(7 \%$ vs. $45 \%$; $\mathrm{P}<0.01$ ). Moreover, patients who had an indwelling catheter for more than 48 hours had a significantly higher rate of bladder infection (35\%) than patients who were straight catheterized and/or who had an indwelling catheter for fewer than 48 hours $(6 \%, \mathrm{P}<0.01)(50)$.

van den Brand et al. (60) performed a prospective RCT to determine whether an indwelling catheter for 48 hours or intermittent catheterization leads to less postoperative bacteriuria or a UTI with a single dose of cefazolin prophylaxis in primary hip and knee arthroplasties. In their protocol, patients received 48 hours of IV prophylactic cefazolin during the postoperative period. Patients who had an indwelling catheter in place after the IV antibiotics were completed were treated with oral antibiotic prophylaxis (nitrofurantoin) until catheter removal. Of the 99 patients who completed the study, 14 patients ( 5 men, 9 women) developed postoperative bacteriuria. The indwelling catheter group had a bacteriuria rate of $24 \%$ (11/46) compared with $6 \%(3 / 53)$ in the intermittent catheterization group $(P=0.018)(60)$.

Similar findings were reported by Oishi et al. (61), who reviewed 95 consecutive patients who had been managed with either an indwelling catheter (72 hours) or intermittent catheterization. Patients who were treated with an indwelling catheter had significantly lower incidences of urinary retention (7\% vs. $84 \%$ respectively; $\mathrm{P}<0.005$ ) and bladder distension ( $7 \%$ vs. $41 \%$; $\mathrm{P}<0.005$ ) than those who were treated with straight catheterization. While not statistically significant, though no patient in the indwelling catheter group developed infection, in the intermittent catheterization group one patient $(2 \%)$ had bacteriuria and one patient $(2 \%)$ had a UTI $(\mathrm{P}>0.1)(61)$.

Koulouvaris et al. (62) performed a retrospective case control study to determine whether a treated preoperative or postoperative UTI or asymptomatic bacteriuria increases the risk of deep PJI and whether the organisms are the same for the UTI and PJI. The authors matched 58 patients who had wound infections with 58 patients who did not develop wound infection based on age, gender, surgeon, joint, year of surgery, and length of follow-up. The authors found no association between preoperative UTI and wound infection (OR 0.34; 95\% CI 0.086 - 1.357, 
Parvizi J et al.

$\mathrm{P}=0.13)$, and no association between postoperative UTI and wound infection (OR 4.22; 95\% CI 0.46-38.9, $\mathrm{P}=0.20$ ). Only one patient had the same bacteria (E. faecalis) cultured in the urine and the wound.

In a survey of the members of the American Society of Breast Surgeons regarding the use of perioperative antibiotics for breast operations requiring drains, respondents continued antibiotic prophylaxis for 2 - 7 days or until all drains were removed (38\% and 39\%, respectively) in cases without reconstruction, while in reconstruction cases 33\% of respondents continued antibiotic prophylaxis for 2 - 7 days or until all drains were removed (63). A similar study surveying the American and Canadian societies of plastic surgeons regarding drain use and perioperative antibiotic prophylaxis in cases of breast reconstruction found that $72 \%$ of plastic surgeons prescribed postoperative outpatient antibiotics in reconstruction patients with drains, with $46 \%$ continuing antibiotics until drains were removed (64).

\section{Conclusions}

Question 6: What are the indications for administration of vancomycin?

Consensus: Vancomycin should be considered for patients who are current MRSA carriers or have anaphylactic allergy to penicillins.

Consideration should be given to screening high risk patients such as:

- Patients in regions with a high prevalence of MRSA.

- Institutionalized patients (nursing home residents, dialysis-dependent patients, and those who have been in the intensive care unit).

- Healthcare workers.

Question 7: Is there evidence to support the routine use of vancomycin for preoperative prophylaxis?

Consensus: No. Routine use of vancomycin for preoperative prophylaxis is not recommended.

Delegate Vote: agree: 93\%, disagree: 6\%, abstain: $1 \%$ (strong consensus)

Question 8: Is there a role for routine prophylactic use of dual antibiotics (cephalosporins and aminoglycosides or cephalosporins and vancomycin)?

Consensus: Routine prophylactic use of dual antibiotics is not recommended.

Question 9: What should be the antibiotic of choice for patients with abnormal urinary screening and/or an indwelling urinary catheter?

Consensus: The presence of urinary tract symptoms should trigger urinary screening prior to TJA. Asymptomatic patients with bacteriuria may safely undergo TJA provided that routine prophylactic antibiotics are administered. Patients with acute urinary tract infections (UTI) need to be treated prior to elective arthroplasty

Question 10: Should the preoperative antibiotic choice be different in patients who have previously been treated for another joint infection?
Consensus: The type of preoperative antibiotic administered to a patient with prior septic arthritis or PJI should cover the previous infecting organism of the same joint. In these patients, we recommend the use of antibiotic-impregnated cement, if a cemented component is utilized.

Question 11: Should postoperative antibiotics be continued while a urinary catheter or surgical drain remains in place?

Consensus: No. There is no evidence to support the support the continued use of postoperative antibiotics when urinary catheter or surgical drains are in place. Urinary catheters and surgical drains should be removed as soon as safely possible.

\section{References}

1. Advisory statement. Recommendations for the use of intravenous antibiotic prophylaxis in primary total joint arthroplasty.. Available from: http://www.aaos.org/about/papers/advistmt/1027.asp.

2. Muto CA, Jernigan JA, Ostrowsky BE, Richet HM, Jarvis WR, Boyce $\mathrm{JM}$, et al. SHEA guideline for preventing nosocomial transmission of multidrug-resistant strains of Staphylococcus aureus and enterococcus. Infect Control Hosp Epidemiol. 2003;24(5):362-86.

3. Hawn MT, Richman JS, Vick CC, Deierhoi RJ, Graham LA, Henderson WG, et al. Timing of surgical antibiotic prophylaxis and the risk of surgical site infection. JAMA Surg. 2013;148(7):649-57.

4. Cantoni L, Glauser MP, Bille J. Comparative efficacy of daptomycin, vancomycin, and cloxacillin for the treatment of Staphylococcus aureus endocarditis in rats and role of test conditions in this determination. Antimicrob Agents Chemother. 1990;34(12):2348-53.

5. Patel M, Kumar RA, Stamm AM, Hoesley CJ, Moser SA, Waites KB USA300 genotype community-associated methicillin-resistant Staphylococcus aureus as a cause of surgical site infections. JClin Microbiol. 2007;45(10):3431-3.

6. Manian FA, Griesnauer S. Community-associated methicillinresistant Staphylococcus aureus (MRSA) is replacing traditional health care-associated MRSA strains in surgical-site infections among inpatients. Clin Infect Dis. 2008;47(3):434-5.

7. US Department of health and human services. Recommendations of the Hospital Infection Control Practices Advisory Committee (HICPAC). MMWR Recomm Rep. 1995;44(RR-12):1-13.

8. Hiramatsu K, Aritaka N, Hanaki H, Kawasaki S, Hosoda Y, Hori S, et al. Dissemination in Japanese hospitals of strains of Staphylococcus aureus heterogeneously resistant to vancomycin. Lancet. 1997;350(9092):1670-3.

9. Michel M, Gutmann L. Methicillin-resistant Staphylococcus aureus and vancomycin-resistant enterococci: therapeutic realities and possibilities. Lancet. 1997;349(9069):1901-6.

10. Fulkerson E, Valle CJ, Wise B, Walsh M, Preston C, Di Cesare PE. Antibiotic susceptibility of bacteria infecting total joint arthroplasty sites.J Bone Joint Surg Am. 2006;88(6):1231-7.

11. UK Health protection agency. Surgical Site Infection surveillance service. Protocol for surveillance of surgical site infection. Available from: http://www.hpa.org/uk/we/HPAwebFile/ HPAweb_C/1194947388966.

12. Meehan J, Jamali AA, Nguyen H. Prophylactic antibiotics in hip and knee arthroplasty. J Bone Joint Surg Am. 2009;91(10):2480-90.

13. Wiesel BB, Esterhai JL. Prophylaxis of musculoskeletal infections. In: JC , JTM editors. Musculosketal Infections.. New York: Marcel Dekker; 2003. pp. 115-29.

14. Merrer J, Desbouchages L, Serazin V, Razafimamonjy J, Pauthier F, Leneveu M. Comparison of routine prophylaxis with vancomycin or cefazolin for femoral neck fracture surgery: microbiological and clinical outcomes. Infect Control Hosp Epidemiol. 2006;27(12):1366-71.

15. Cranny G, Elliott R, Weatherly H, Chambers D, Hawkins N, Myers 
L, et al. A systematic review and economic model of switching from non-glycopeptide to glycopeptide antibiotic prophylaxis for surgery. Health Technol Assess. 2008;12(1):iii-iv-1-147.

16. Bolon MK, Morlote M, Weber SG, Koplan B, Carmeli Y, Wright SB. Glycopeptides are no more effective than beta-lactam agents for prevention of surgical site infection after cardiac surgery: a meta-analysis. Clin Infect Dis. 2004;38(10):1357-63.

17. Mangram AJ, Horan TC, Pearson ML, Silver LC, Jarvis WR. Guideline for prevention of surgical site infection, 1999. Hospital Infection Control Practices Advisory Committee. Infect Control Hosp Epidemiol. 1999;20(4):250-78

18. Tacconelli E, Cataldo MA, Albanese A, Tumbarello M, Arduini E, Spanu T, et al. Vancomycin versus cefazolin prophylaxis for cerebrospinal shunt placement in a hospital with a high prevalence of meticillin-resistant Staphylococcus aureus. J Hosp Infect. 2008;69(4):337-44.

19. Finkelstein R, Rabino G, Mashiah T, Bar-El Y, Adler Z, Kertzman V, et al. Vancomycin versus cefazolin prophylaxis for cardiac surgery in the setting of a high prevalence of methicillin-resistant staphylococcal infections. J Thorac Cardiovasc Surg. 2002;123(2):326-32.

20. Garey KW, Lai D, Dao-Tran TK, Gentry LO, Hwang LY, Davis BR. Interrupted time series analysis of vancomycin compared to cefuroxime for surgical prophylaxis in patients undergoing cardiac surgery. Antimicrob Agents Chemother. 2008;52(2):446-51.

21. Spelman D, Harrington G, Russo P, Wesselingh S. Clinical, microbiological, and economic benefit of a change in antibiotic prophylaxis for cardiac surgery. Infect Control Hosp Epidemiol. 2002;23(7):402-4.

22. Smith EB, Wynne R, Joshi A, Liu H, Good RP. Is it time to include vancomycin for routine perioperative antibiotic prophylaxis in total joint arthroplasty patients? J Arthroplasty. 2012;27(8 Suppl):55-60.

23. Bull AL, Worth LJ, Richards MJ. Impact of vancomycin surgical antibiotic prophylaxis on the development of methicillin-sensitive staphylococcus aureus surgical site infections: report from Australian Surveillance Data (VICNISS). Ann Surg. 2012;256(6):1089-92.

24. Miller LG, McKinnell JA, Vollmer ME, Spellberg B. Impact of methicillin-resistant Staphylococcus aureus prevalence among S. aureus isolates on surgical site infection risk after coronary artery bypass surgery. Infect Control Hosp Epidemiol. 2011;32(4):342-50.

25. Zanetti G, Giardina R, Platt R. Intraoperative redosing of cefazolin and risk for surgical site infection in cardiac surgery. Emerg Infect Dis. 2001;7(5):828-31.

26. Zanetti G, Goldie SJ, Platt R. Clinical consequences and cost of limiting use of vancomycin for perioperative prophylaxis: example of coronary artery bypass surgery. Emerg Infect Dis. 2001;7(5):820-7.

27. Muralidhar B, Anwar SM, Handa AI, Peto TE, Bowler IC. Prevalence of MRSA in emergency and elective patients admitted to a vascular surgical unit: implications for antibiotic prophylaxis. Eur J Vasc Endovasc Surg. 2006;32(4):402-7.

28. Gemmell CG, Edwards DI, Fraise AP, Gould FK, Ridgway GL, Warren RE, et al. Guidelines for the prophylaxis and treatment of methicillin-resistant Staphylococcus aureus (MRSA) infections in the UK. J Antimicrob Chemother. 2006;57(4):589-608.

29. Elliott RA, Weatherly HL, Hawkins NS, Cranny G, Chambers D, My ers L, et al. An economic model for the prevention of MRSA infections after surgery: non-glycopeptide or glycopeptide antibiotic prophylaxis? Eur J Health Econ. 2010;11(1):57-66.

30. Walsh EE, Greene L, Kirshner R. Sustained reduction in methicillin-resistant Staphylococcus aureus wound infections after cardiothoracic surgery. Arch Intern Med. 2011;171(1):68-73.

31. Dhadwal K, Al-Ruzzeh S, Athanasiou T, Choudhury M, Tekkis P, Vuddamalay P, et al. Comparison of clinical and economic outcomes of two antibiotic prophylaxis regimens for sternal wound infection in high-risk patients following coronary artery bypass grafting surgery: a prospective randomised double-blind controlled trial. Heart. 2007;93(9):1126-33.

32. Patrick S, James C, Ali A, Lawson S, Mary E, Modak A. Vascular surgical antibiotic prophylaxis study (VSAPS). Vasc Endovascular Surg. 2010;44(7):521-8.
33. Sewick A, Makani A, Wu C, O'Donnell J, Baldwin KD, Lee GC Does dual antibiotic prophylaxis better prevent surgical site infections in total joint arthroplasty? Clin Orthop Relat Res 2012;470(10):2702-7.

34. Ritter MA, Barzilauskas CD, Faris PM, Keating EM. Vancomycin prophylaxis and elective total joint arthroplasty. Orthopedics. 1989;12(10):1333-6.

35. Cruess RL, Bickel WS, vonKessler KL. Infections in total hips secondary to a primary source elsewhere. Clin Orthop Relat Res. 1975(106):99-101.

36. Hall AJ. Late infection about a total knee prosthesis. Report of a case secondary to urinary tract infection. J Bone Joint Surg Br. 1974;56(1):144-7.

37. David TS, Vrahas MS. Perioperative lower urinary tract infections and deep sepsis in patients undergoing total joint arthroplasty. Am Acad Orthop Surg. 2000;8(1):66-74.

38. Ritter MA, Fechtman RW. Urinary tract sequelae: possible influence on joint infections following total joint replacement. Orthopedics. 1987;10(3):467-9.

39. Wymenga AB, van Horn JR, Theeuwes A, Muytjens HL, Slooff TJ Perioperative factors associated with septic arthritis after arthroplasty. Prospective multicenter study of 362 knee and 2,651 hip operations. Acta Orthop Scand. 1992;63(6):665-71.

40. Glynn MK, Sheehan JM. The significance of asymptomatic bacteriuria in patients undergoing hip/knee arthroplasty. Clin Orthop Relat Res. 1984(185):151-4.

41. Waterhouse N, Beaumont AR, Murray K, Staniforth P, Stone MH Urinary retention after total hip replacement. A prospective study. J Bone Joint Surg Br. 1987;69(1):64-6.

42. Walton JK, Robinson RG. An analysis of a male population having total hip replacement with regard to urological assessment and post-operative urinary retention. Br JUrol.1982;54(5):519-21.

43. Drekonja DM, Rector TS, Cutting A, Johnson JR. Urinary tract infection in male veterans: treatment patterns and outcomes. JAMA Intern Med. 2013;173(1):62-8.

44. Irvine R, Johnson BJ, Amstutz HC. The relationship of genitourinary tract procedures and deep sepsis after total hip replacements. Surg Gynecol Obstet. 1974;139(5):701-6.

45. Garibaldi RA, Burke JP, Dickman ML, Smith CB. Factors predisposing to bacteriuria during indwelling urethral catheterization. $N$ Engl J Med. 1974;291(5):215-9.

46. Kunin CM, McCormack RC. Prevention of catheter-induced urinary-tract infections by sterile closed drainage. $N$ Engl J Med. 1966;274(21):1155-61.

47. Jerry GJ, Rand JA, Ilstrup D. Old sepsis prior to total knee arthroplasty. Clin Orthop Relat Res. 1988(236):135-40.

48. Lee GC, Pagnano MW, Hanssen AD. Total knee arthroplasty after prior bone or joint sepsis about the knee. Clin Orthop Relat Res. 2002(404):226-31.

49. Larson AN, Hanssen AD, Cass JR. Does prior infection alter the outcome of TKA after tibial plateau fracture? Clin Orthop Relat Res. 2009;467(7):1793-9.

50. Michelson JD, Lotke PA, Steinberg ME. Urinary-bladder management after total joint-replacement surgery. $N$ Engl J Med. 1988;319(6):321-6.

51. Martinez OV, Civetta JM, Anderson K, Roger S, Murtha M, Malinin TI. Bacteriuria in the catheterized surgical intensive care patient. Crit Care Med.1986;14(3):188-91.

52. Schaeffer AJ. Catheter-associated bacteriuria. Urol Clin North Am 1986;13(4):735-47.

53. Skelly JM, Guyatt GH, Kalbfleisch R, Singer J, Winter L. Management of urinary retention after surgical repair of hip fracture. CMAJ. 1992;146(7):1185-9.

54. Donovan TL, Gordon RO, Nagel DA. Urinary infections in total hip arthroplasty. Influences of prophylactic cephalosporins and catheterization. J Bone Joint Surg Am. 1976;58(8):1134-7.

55. Fitzgerald RJ, Nolan DR, Ilstrup DM, Van Scoy RE, Washington J2, Coventry MB. Deep wound sepsis following total hip arthroplasty. JBone Joint Surg Am. 1977;59(7):847-55.

56. Surin VV, Sundholm K, Backman L. Infection after total hip replacement. With special reference to a discharge from the wound.J Bone Joint Surg Br. 1983;65(4):412-8. 
57. Felippe WA, Werneck GL, Santoro-Lopes G. Surgical site infection among women discharged with a drain in situ after breast cancer surgery. World J Surg. 2007;31(12):2293-9.

58. Lanier ST, Wang ED, Chen JJ, Arora BP, Katz SM, Gelfand MA, et al. The effect of acellular dermal matrix use on complication rates in tissue expander/implant breast reconstruction. Ann Plast Surg. 2010;64(5):674-8.

59. Sorensen AI, Sorensen TS. Bacterial growth on suction drain tips. Prospective study of 489 clean orthopedic operations. Acta Orthop Scand. 1991;62(5):451-4.

60. van den Brand IC, Castelein RM. Total joint arthroplasty and incidence of postoperative bacteriuria with an indwelling catheter or intermittent catheterization with one-dose antibiotic prophylaxis: a prospective randomized trial. J Arthroplasty. 2001;16(7):850-5.

61. Oishi CS, Williams VJ, Hanson PB, Schneider JE, Colwell CJ, Walker
RH. Perioperative bladder management after primary total hip arthroplasty. J Arthroplasty. 1995;10(6):732-6.

62. Koulouvaris P, Sculco P, Finerty E, Sculco T, Sharrock NE. Relationship between perioperative urinary tract infection and deep infection after joint arthroplasty. Clin Orthop Relat Res. 2009;467(7):1859-67.

63. Brahmbhatt RD, Huebner M, Scow JS, Harmsen WS, Boughey JC, Harris AM, et al. National practice patterns in preoperative and postoperative antibiotic prophylaxis in breast procedures requiring drains: survey of the American Society of Breast Surgeons. Ann Surg Oncol. 2012;19(10):3205-11.

64. Phillips BT, Wang ED, Mirrer J, Lanier ST, Khan SU, Dagum AB, et al. Current practice among plastic surgeons of antibiotic prophylaxis and closed-suction drains in breast reconstruction: experience, evidence, and implications for postoperative care. Ann Plast Surg. 2011;66(5):460-5. 